\title{
Earthquake frequency and moment magnitude relations for mainshocks, foreshocks and aftershocks: Theoretical $b$ values
}

Department of Earth and Space Sciences, Graduate School of Science. Osaka University. I-I Machikaneyama, Toyonaka, Osaka 560 , Japan

The Gutenberg-Richter relation, $\log n=a-b M$, where $n$ is the cumulative number of earthquakes greater than the magnitude, $M$ and $a$ and $b$ are constants determined empirically, is discussed using a mathematical model of a fault and the moment magnitude, $M_{W}$. A simple power law of $M_{0}=k n^{l}$ which relates the earthquake moment, $M_{0}$ and frequency, $n$ was discussed and the meanings of constants $k$ and $l$ were derived. The relation of $M_{0}=\Delta \sigma$ (LA) using the stress drop, $\Delta \sigma$, fault length, $L$ and area of a fault plane, $A$, gives $M_{O}=\Delta \sigma L^{3}$ and $M_{0}=\Delta \sigma h L^{2}$ for two cases of $A=L^{2}$ and $A=L h$, respectively, where the depth of the brittle-ductile transition laver, $h=2 x$ $10^{4} \mathrm{~m}$ and $\Delta \sigma=5 \times 10^{6} \mathrm{~N} / \mathrm{m}^{2}$ are assumed. The frequency-magnitude relation is obtained considering different rupture mechanisms of earthquakes. Deviation of the empirical Gutenberg-Richter relation at a large magnitude is interpreted as the effect of the seismogenic layer in earth interior. Theoretical analysis from assumption of different rupture mechanisms indicated varied $b$ values for small and large events, as well as the different types of earthquakes. Mainshocks gave b of 1.0 and 0.75 , respectively for small and large events. The $b$ values of 0.75 and 0.50 for aftershocks and 0.50 and 0.375 for foreshocks were also obtained. Some results from both statistic calibration of earthquake catalogue and experiments were in agreement with our predicted values.

\section{Introduction}

The Gutenberg-Richter relation (GRR). $\log n=a-b M$. where $n$ is the cumulative earthquakes greater than the magnitude. $M$ and $a$ and $b$ are constants has been used for a long time as an empirical relation of frequency and magnitude of earthquakes (Gutenberg and Richter, 1954). It has been interpreted as a self-similarity elfect of earthquakes (Bak and Tang, 1989: Ito and Matsuzaki, 1990). The estimation of recurrence interval hased on this relation is also useful for scismic hazard assessment (Turcotte, 1992; Marrett, 1994). However, its failing in prediction of high magnitude made some researchers doubt its validity in a broau magnitude (Main, 1992: Hamilton and McCloskey, 1997).

Recently, various interpretations of the frequency-magnitude relation of large events are made. Although there is consensus that the finite thickness of the scismogenic layer changes the scaling of large earthquakes (except Sornctic. el al.. 1996), the obtained $b$ values are in controversy (Rundle. 1989: Pacheco et al.. 1992: Main and Berton. 1986: Main. 1992; Hamilton and McCloskey, 1997).

Different interpretations are consistent with different models of large earthquakes, such as the $L$-model (Scholy, 1982) in which the slip and fault length will keep increasing with earthquake size and the $W$-model (Romanowicz, 1992: Romanowicz and Rundle. 1993) in which both slip and width of fault saturate for large events. However, the controversy of $b$ values from each evidence support leads to the confusion in understanding the physical insight of the frequencymagnitude relation and $b$ values.

A mathematical fault model, moment magnitude, $M_{w}$. as well as a power law of $M_{0}=k \mathrm{~m}^{\prime}$. where $M_{0}$ is earthquake moment and $k$ and $l$ are constants, are discussed in this paper. GRR is derived considering the different rupture mechanisms of small and large earthquakes (including volcanic eruptions. foreshocks mainshocks and aftershocks). The physical meaning of $b$ values for different types of earthquakes is analyzed. The obtained theoretical $b$ values are used to predict the $b$ values either for carthquake catalogue or for simulated experiments.

\section{The relation of scaling law and power law}

\section{Power law of frequency-moment relation}

GRR has been investigated by a number of researchers and a value of $b=1$ is almost always found either from observation or from theoretical analysis (Fukalo and Furumoto. 1985: Rundle, 1989). However, in order to fit the relation. different magnitudes were used by various investigators. e.g. surface-wave magnitude. $M$, for latrer events. body-wave magnitude. $m_{\mathrm{b}}$ for smaller ones and local magnitude. $M_{\mathrm{L}}$ for cven smaller events. This may lead to confusion in understanding the physical meaning of $b$ values.

The relation of earthquake frequency and moment is physically more meaninglul than that of frequency and magnitude 6 Okal and Romanowic\% 1994). Such pọwer law can be expressed as.

$$
M_{0}=k n^{l}
$$

Because the concept of magnitude has been used for a long time not only among seismologists but also by the news media. it would be convenient to quantify the sice of earthquake in terms of a mannitude scale. Different definitions of magnitude may be useful for researchers, however, lead to confusion for common audience. Therefore, we use $M_{\mathrm{W}}$ which is a definition by $M_{0}$ as following (Kanamori. 1978),

$$
M_{\mathrm{W}}=\left(\log M_{9}-9.1\right) / 1.5
$$

Note that $S I$ unit is used in all discussions here.

We assumed the magnitude, $M=M_{W}$ in following discussions not only for simplicity but also for more quantitative physical meaning. Therefore, from Eq. (1) Eq. (2) and the cmpirical frequency- 
magnitude relation (theoretical analysis will be undertaken in next section).

$$
\log n=a-b M
$$

constants of $k$ and $l$ could be obtained als. $k=10^{9.1+1.5(t /)}$ and $l=$ $-1.5 / b$, respectively.

\section{General scaling law}

Considering the similarity of carthquakes, there should be a general scaling law correlating some physical quantity, $X$ and $M_{0}$, i. e.,

$$
M_{0}=k X^{\prime}
$$

The frequency-moment relation, Eq. (1) is just a special case of the general scaling law. Eq. (4), when $X=n$. Besides $n$, many other quantities, such as rupture length, dislocation, rupture area, seismic energy, precursor time. and so on, were also found to follow some scaling relations from observing data (Rikitake, 1975, 1986; Dambara. 1981). Theoretical analysis was undertaken based on a two-dimensional fault model (Chen and Chen, 1989; Chen and Bai, 1991). However, neither empirical approach nor theoretical analysis dealt with the effect of small and large earthquakes, e. g., Dambara (1981) admitted his empirical relation could not predict observing ditta well for large events. The detailed analysis of the general scaling law for various quantities will be undertaken elsewhere.

\section{Mathematical model of a fault for small and large earthquakes}

The earthquake moment, $M_{0}$ defined as $M_{0}=\mu D A$ in a mathematical model of a fault. using the rigidity of the earth, $\mu$, displacement of a fault, $D$ and the area of a fault plane, $A$. By using stress drop, $\Delta \sigma=\mu$ $(I) / L), M_{0}$ can be re-written as

$$
\left.M_{0}=\mu D A=\mu(D) / L\right) L A=\Delta \sigma(L A)
$$

Considering the existence of brittle-ductile transition layer (depth $=h$ ) in earth interior (Pacheco et al., 1992), it"s more plausible to discuss the scaling law for two cases: small $(L<h)$ and large earthquakes $(L>h)$. The critical moment magnitude, $M_{\mathrm{c}}$ was estimated from Eqs. (2) and (4), using $\Delta \sigma$ and $h$. For simplicity, we didn't apply the empirical relation of fault dimension (Geller, 1976). Hence, $M_{0}$ for $A=L^{2}$ (small events) and $A=L h$ (large events) is given as

and

$$
M_{0}=L^{3} \Delta \sigma
$$

$$
M_{0}=L^{2} h \Delta \sigma
$$

A tentative assumption of $h=L=2 \times 10^{4} \mathrm{~m}$ and $\Delta \sigma=5 \times 10^{6}$ $\mathrm{N} / \mathrm{m}^{2}$ leads to the critical moment magnitude, $M_{\mathrm{c}}=7.0$. Of course, $\Delta \sigma$ would vary either for various types of earthquakes or for different regions (Kanamori and Anderson, 1975) and the critical depth $h$ would change from place to place. Therefore, different thresholds were obtained by various authors, e. g., a critical fault width (equivalent to the depth here) of $30 \mathrm{~km}$ (Okal and Romanowicz, 1994), a break point of magnitude of 6.0-7.5 (Main, 1992: Pacheco et al., 1992). For simplicity, our assumption is based on the average values of $h$ and $\Delta \sigma$. Logarithmic definition of $M$ indicates $M_{\mathrm{c}}=7.0 \pm 0.6$ for the factor 2 difference of $h$ and $M_{c}=7.0 \pm 0.2$ for the factor 2 difference of $\Delta \sigma$, respectively.

\section{Gutenberg and Richter relation}

Various investigators described the GRR from the fractal theory or other rupture hypothesis in elucidation of $b$ values for mainshocks. We estimate the $b$ values theoretically for two cases of small $(M<$ $7.0)$ and large $(M>7.0)$ earthquakes (including foreshocks and aftershocks), based on the relation between rupture and the length of a fault, $L$.

\section{Three dimensional ruptures: bulk ruptures}

Uniform materials give the size with a diameter $L$ and the frequency $n$ reciprocally proportional to volume. Asteroids, planets and craters satisfy this relation (Utsu, 1985, pp. 138-139). If this is the case for earthquakes, Equations (2), (6) and (7) lead to

and

$$
M<7.0: n \propto L^{-3} \propto M_{0}^{-1} \text {, hence, } b=1.5
$$

$$
M>7.0: n \propto L^{-2} h^{-1} \propto M_{0} 0^{-1} \text {, hence. } b=1.5 \text {. }
$$

These do not agree with the GRR with $b$ of about 1 . However, they are consistent with $b$ values of about 1.5 for compression on very heterogeneous materials, e. g., granular pumice, in the elastic shocks accompanying the fracture (Mogi, 1962). Thus. bulk ruptures would lead to $b=1.5$. The higher $b$ values of volcanic earthquakes, e. g., $b=1.5$, may be interpreted as the effect of three dimensional ruptures.

\section{Two dimensional ruptures: mainshocks}

A main shock is due to the movement of a fault. It's considered as a two dimensional rupture. Hence, the frequency, $n$ would be reciprocally proportional to $A$ and lead to

and

$$
\left.M<7.0: n \propto 1 / A \propto L^{-2} \propto M_{0}\right)^{-2 / 3} \text {, hence, } b=1.0
$$

$$
M>7.0: n \propto 1 / A \propto L^{-1} h^{-1} \propto M_{0}^{-1 / 2} \text {, hence, } b=0.75 \text {. }
$$

Just similar to many previous investigations, a unity $b$ value in GRR was obtained here. However, this is only for small earthquakes. In case of large events, a small $b$ value of 0.75 appeared. Such decrease of $b$ value at large magnitude scale was consistent with some recent interpretations (Main and Burton, 1986; Main, 1992; Cowie et al., 1993; Hamilton and McCloskey. 1997), but contradicted with the result of $b=1.5$ (Rundle, 1989; Pacheco et al., 1992). Such controversy may be due to the different understanding of rupture mechanism (also see the section of discussion).

Empirical $b$ values are varied with regions, e. g., Gutenberg and Richter (1954) obtained $b=0.88$ from earthquakes in south California, an investigation of earthquakes around Japan during 1965-1974 gave $b=0.936$ (Utsu, 1985, pp. 134). These $b$ values may be due to their attempt of fitting both small and large earthquakes within one line (see discussion section). Experiments also indicated the existence of varied $b$ values (Mogi, 1962; Scholz, 1968). As suggested from experimental results, regional variations of $b$ values may reflect the variations of stress state there (Scholz, 1968).

\section{One dimensional ruptures: aftershocks}

Aftershocks occur by the one dimensional ruptures, i. e., along the edge of the fault plane. This may be due to the pre-ruptured effect by the mainshock. Hence, $n \propto L^{-1}$ may be assumed in case of aftershocks and leads to

and

$$
\left.M<7.0: n \propto \mathrm{L}^{-1} \propto M_{0}\right)^{-1 / 3} \text {, hence, } b=0.50
$$

$$
\mathrm{M}>7.0: n \propto L^{-1} \propto M_{0}^{-1 / 2} \text {, hence, } b=0.75 \text {. }
$$

Above analysis indicated that small $b$ values are found for aftershocks, comparing with those of mainshocks. It would be plausible that $b$ values can reflect the different characteristics of carthquakes. An example of aftershocks of Mikawa earthyuake (Jan. 13, 1945, M 6.8, Japan) gave $b=0.75$ (Utsu, 1985, pp. 180).

\section{Ensemble of small element ruptures: foreshocks}

If the fracture occurs at a fixed number of sites in a fault plane, with a small fracture length of $L^{\prime}$ and the seismic waves are detected simultaneously at an observation point, a relation of $n \propto L^{-1 / 2}$ would appear as described in the following.

Considering the energy of ruptures is due to the small asperity fractures, the earthquake moment of a foreshock, $M_{0}$ would be a sum of those of small fractures, namely, $M_{\mathrm{Of}}=\sum \mu_{\mathrm{i}} D_{\mathrm{i}} A_{\mathrm{i}}{ }^{\prime}=\sum \Delta \sigma_{\mathrm{i}}$ $\left(L_{\mathrm{i}} A_{\mathrm{i}}{ }^{\prime}\right.$, where $\mu_{\mathrm{i}}, D_{\mathrm{i}}, L_{\mathrm{i}}, A_{\mathrm{i}}$ and $\Delta \sigma_{\mathrm{i}}$ are rigidity of medium, displacement, length of rupture, fracture area and stress drop for each 
small fracture respectively. On the other hand, the moment of a foreshock can be written effectively as $M_{0 f}=\Delta \sigma\left(L_{f} A_{f}\right)$, using average stress drop, $\Delta \sigma$, effective length, $L_{i}$ and fault area, $A_{j}$. The foreshock propagates the earthquake volume with the time $\tau \propto L_{1} / \beta$, where $\beta$ is the velocity of $S$-waves. The power, i. e., the energy of seismic waves per unit time as $E_{f} / \tau$, which is proportional to the square of the amplitude, $I_{1}$, is expressed as a superposition of seismic waves with random phases. If $N$ is the total number of small fractures for a certain foreshock, the statistical calibration gives $I_{\mathrm{f}}=N^{1 / 2} l_{\mathrm{i}}{ }^{\circ} \propto\left(E_{\mathrm{f}} / \tau\right)^{1 / 2}$, where $I_{\mathrm{i}}{ }^{\prime}$ is the amplitude of each small fracture. Using $E_{f} \propto M_{O f}$. two cases of $A_{\mathrm{f}}=L_{\mathrm{f}}{ }^{2}$ and $A_{\mathrm{f}}=L_{\mathrm{f}} h$ may lead to $I_{\mathrm{f}} \propto L_{\mathrm{f}}$ and $I_{\mathrm{f}} \propto L_{\mathrm{f}}{ }^{1 / 2}$, respectively. Therefore, there are

$$
M<7.0: n \propto I_{f^{-1}}^{-1} \propto L_{\mathrm{f}}^{-1} \propto M_{0}^{-1 / 3} \text {, hence, } b=0.50
$$

and

$$
M>7.0: n \propto I_{1^{-1}} \propto L_{1}^{-1 / 2} \propto M_{0}^{-1 / 4} \text {, hence, } b=0.375 \text {. }
$$

We summarized the $b$ values in GRR and the power law for mainshocks, foreshocks and aftershocks, as well as bulk ruptures, in Table 1

Table 1 Theoretical $b$ values in frequency $(n)$ and magnitude $(M)$ relation, $\log n=a-b M$, using moment magnitude, $M_{W}=M . M_{0}$ is

\begin{tabular}{|c|c|c|c|c|}
\hline $\begin{array}{l}\text { Relation of } \\
n \text { and } M_{0}\end{array}$ & $b$ & $M$ & $\begin{array}{l}\text { Rupture } \\
\text { type }\end{array}$ & $\begin{array}{l}\text { Type of } \\
\text { earthquake }\end{array}$ \\
\hline$n \propto L^{3}$ & 15 & $<7.0$ & bulk & volcanic \\
\hline$\propto M_{0}^{-1}$ & & $>7.0$ & & eruptions \\
\hline $\begin{aligned} n & \propto A^{-1} \propto L^{-2} \\
& \propto M_{0}^{-2 / 3}\end{aligned}$ & 1.0 & $<0.7$ & planar & mainshocks \\
\hline $\begin{aligned} n \propto A^{-1} \propto L^{-1} & \propto M_{0}^{-1 / 2}\end{aligned}$ & 0.75 & $>7.0$ & planar & mainshocks \\
\hline $\begin{aligned} n & \propto L^{-1} \\
& \propto M_{0}{ }^{1 / 3}\end{aligned}$ & 0.50 & $<7.0$ & line & aftershocks \\
\hline $\begin{aligned} n & \propto L^{-1} \\
& \propto M_{0}^{-1 / 2}\end{aligned}$ & 0.75 & $>7.0$ & line & aftershocks \\
\hline $\begin{array}{l}n \propto L^{-1} \\
\propto M_{0}^{-1 / 3}\end{array}$ & 0.50 & $<7.0$ & $\begin{array}{l}\text { ensemble } \\
\text { points }\end{array}$ & foreshocks \\
\hline $\begin{aligned} n & \propto L^{-1 / 2} \\
& \propto M_{0}^{-1 / 4}\end{aligned}$ & 0.375 & $>7.0$ & $\begin{array}{l}\text { ensemble } \\
\text { points }\end{array}$ & foreshocks \\
\hline
\end{tabular}
earthquake moment and $L$ is the length of a fault.

\section{Discussion}

\section{Controversy of $b$ values at large magnitude}

As investigated by a number of authors, a consensus that breakdown GRR at high magnitude was made. There is also an agreement that the existence of seismogenic layers affects the scaling law of large earthquakes. However. different models lead to a controversy of $b$ values for large events. Each seems to have evidence either from model analysis or from earthquake catalogue or simulation experiment.

It's not easy to judge which interpretation is more reasonable because of the limited amount of large earthquakes. Our results seem to support the stand with a decrease of $b$ values at high magnitude (Main and Burton, 1986; Main, 1992; Cowie et al., 1993; Hamilton and McCloskey, 1997). However, Rundle (1989) derived $b=1.5$ for large events, which was supported by the analysis of a worldwide catalogue of shallow earthquakes (Pacheco et al. 1992).

Note that in our analysis, the average values of $h=2 \times 10^{4} \mathrm{~m}$ and $\Delta \sigma=5 \times 10^{6} \mathrm{~N} / \mathrm{m}^{2}$ were used in the estimation of $M_{\mathrm{c}}$. This assumes a $L$-model implicitly as using a constant stress change for all earthquakes with $\Delta \sigma=\mu D / L$. However, the catalogue used by Pacheco et al. includes all earthquakes $(M \geq 7.0$ and depth $\leq 60 \mathrm{~km}$ ) during 1900-1989. We will not discuss the error of estimation magnitude for such a long period. Instead, such controversy might be due to the earthquake catalogue they used. Shallow earthquakes are often defined as those with a depth less than $60 \mathrm{~km}$ in textbook of seis- mology. However, the seismogenic layer is much shallower than this threshold, i. e., about $5-15 \mathrm{~km}$ for transform faults. The consensus of the seismogenic layer effect indicates that it would be more plausible to use much shallower earthquakes. i. e., depth $\leq 20 \mathrm{~km}$ an we assumed, or $30 \mathrm{~km}$ as Okal and Romanowicz (1994) suggested. If this is the case, a different variation characteristics of $b$ values for large events (comparing with the result of Pacheco et al., 1992) would be found. Therefore, it's necessary to re-examine the existence of such contradiction. On the other hand, if we deal with shallow earthquakes as defined in seismology, i. e., to a depth of $60 \mathrm{~km}$. it would be necessary to re-estimate $b$ values as we did in the section "two dimentional ruptures: mainshocks". In this case. using planar rupture as $n \propto L^{-2}$ and $M_{0} \propto L^{2}$ for large carthquakes, it would lead to $\mathrm{n} \propto M_{0}{ }^{-1}$. hence, $b=1.5$ is obtained. just the same as the result of Pacheco et al. (1992).

\section{Critical magnitude and moment magnitude}

The above analyses suggested the variation of $b$ values as an effect of different rupture mechanisms. We constricted the discussions in case of shallow earthquakes, without consideration of dip behavior of a fault for simplicity. The thickness of the seismogenic zone should play an important role in rupture models of small and large earthquakes. The critical magnitude. $M_{c}$ is determined by the tectonic stress, as well as the seismogenic layer. Therefore, one should not expect a worldwide standard crossover magnitude. This analysis may explain the observed data which have various $M_{\mathrm{c}}$ in different regions. The difference in $M_{\mathrm{c}}$ can be estimated as described in section "Mathematical model of a fault". In our case, the average values. i. e., $h=2 \times 10^{+} \mathrm{m}$ and $\Delta \sigma=5 \times 10^{6} \mathrm{~N} / \mathrm{m}^{2}$. were assumed to estimate the critical magnitude, $M_{c}$, e. g., $M_{c}=7 .(0)$. This threshold value is consistent with some recent results, i. e., $M_{\mathrm{c}}$ is 6.0-7.5 (Main, 1992: Pacheco et al.. 1992)

It should be noted that different magnitudes are used in GRR by various authors. e. g., the moment magnitude, $M_{\mathrm{W}}$ is used in our discussions. Hence, either the $b$ values or $M_{c}$ should be a little different from those discussions by using other magnitude. However, using the moment magnitude, at least the saturation problem has heen avoided theoretically; furthermore. we can explain the distribution characteristics of all the natural earthquakes (without saturation trouble) after taking two branches into account rather than single line of GRR.

The two branches data fitting would be more plausible than single line fitting in plotting frequency-magnitude relation. Figure 1 is an example of frequency-magnitude plot. Utsu (1985) gave $b=$ 0.936 by one line fitting. We re-examined the original data (Utsu, 1985, Tab. 5.6) and fitted by two branches, giving $b$ values of $1.00 \pm$ 0.01 and $0.68 \pm 0.05$, for small and large earthquakes, respectively,

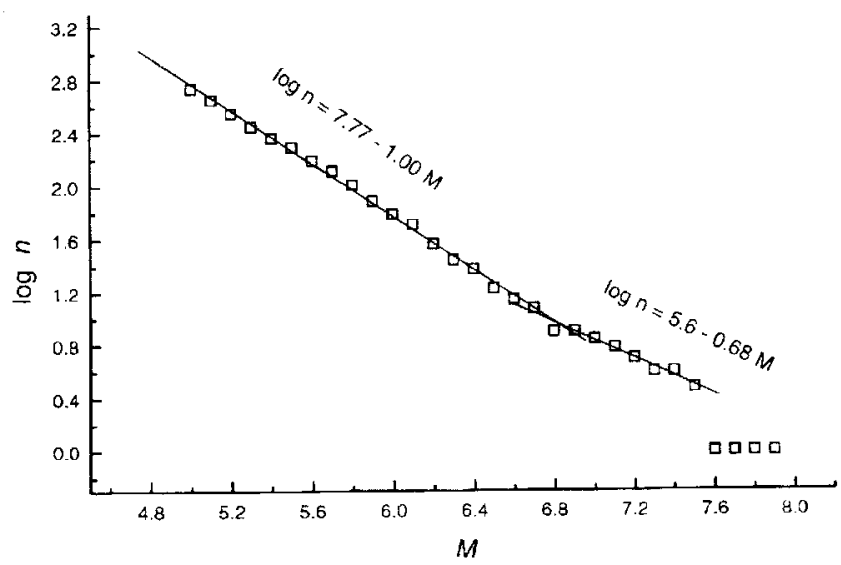

Figure 1 Frequency-magnitude relation of shallow earthquakes during 1965-1974 around Japan. Original data (hollow square) are from Utsu (1985, Tab. 5.6). Rather than single line fitting (Utsu, 1985, Fig. 5.4), two branches are used with a break at $M=$ 6.8 . 
with a break at $M=6.8$. These are in good agreement with our analytical results of $b=1.0$ (small events), $b=0.75$ (large events) and $M_{\mathrm{c}}$ $=7.0$.

Note that we have not taken earthquakes with $M \geq 7.6$ into account because of the bad confidence (only one earthquake with $M$ $=7.9$ occurred within the discussed region and period). Although the application of moment magnitude avoids the saturation problem of earthquake size and makes it possible to extrapolate GRR to high magnitude (even there is a break in the slope), one should not expect to extrapolate such relation to infinity, because of the unreality of expecting an infinite amount of energy be released from the earth's interior (Knopoff and Kagan, 1977; Kagan and Knopoff, 1984; Kagan, 1994).

\section{Conclusion}

A mathematical model of a fault was proposed to produce Gutenberg-Richter relation (GRR). Frequency of earthquakes, $n$ and moment magnitude, $M_{\mathrm{W}}$ were correlated by considering the rupture mechanism and the fault length, $L$. A simple power law of $M_{0}=k n^{1}$ gave constants of $k$ and $l$ as $k=10^{9.1+1.5} \mathrm{a} / \mathrm{b}$ and $l=-1.5 / \mathrm{b}$, respectively using the $b$ values in GRR. The earthquake moment, $M_{0}=\Delta \sigma$ (LA) using the stress drop, $\Delta \sigma$ and area of a fault plane, $A$, gives $M_{0}$ $=\Delta \sigma L^{3}$ and $M_{0}=\Delta \sigma h L^{2}$ for two cases of $A=L^{2}$ and $A=L h$, respectively, where the depth of the brittle-ductile transition layer, $h$ $=2 \times 10^{4} \mathrm{~m}$ and stress drop, $\Delta \sigma=5 \times 10^{6} \mathrm{~N} / \mathrm{m}^{2}$ were assumed. Theoretical analysis from assumption of different rupture mechanisms indicated the existence of varied $b$ values for small and large events. Theoretical $b$ values of different types of earthquakes, such as mainshocks, aftershocks and foreshocks are obtained, consistent with those from both statistic calibration of earthquake catalogue and experiments. Therefore, it's more reasonable to fit the observed data using two branches with a critical magnitude.

\section{References}

Bak, P. and Tang, C., 1989, Earthquakes as a self-organized critical phenomenon. J. Geophys. Res., 94, 15635-15637.

Chen, P. and Chen, P., 1990, Scaling law from two-dimensionall faulting model. Acta Seismol. Sin., 3, 405-420.

Chen, P. and Bai, T., 1992, Quantification relations between the source parameters, Acta Seismol. Sin., 5, 435-446.

Cowie, P. A., Vanneste, C. and Sornette, D., 1993, Statistical physics model for the spatiotemporal evolution of faults, J. Geophys. Res., 98, 21809. 21821.

Dambara, T., 1981, Geodesy and earthquake prediction, in: Rikitake, T. (ed.), Current research in earthquake prediction 1. Center for Academic Publications Japan/Japan Scientific Societies Press, Tokyo, pp. 167-220.

Fukao, Y. and Furumoto, M., 1985, Hierarchy in earthquake size distribution. Phys. Earth Planet. Inter., 37, 149-168.

Geller, R. J., 1976, Scaling relations for earthquake source parameters and magnitudes, Bull. Seismol. Soc. Am., 66, 1501-1523.

Gutenberg, B. and Richter, C. F., 1954, Seismicity of the Earth. Princeton University Press, Princeton, NJ, pp. 310.

Hamilton, T. and McCloskey, J., 1997, Breakdown in power-law scaling in an analogue model of earthquake rupture and stick-slip, Geophys. Res. Lett., 24, 465-468.

Ikeya, M., Takaki, S., Matsumoto, H., Tani, A. and Komatsu, T., 1997. Pulsed charge model of a fault behavior producing seismic electric signals. J. Circuit Systems and Computers, 7 (in press).

Ito, K. and Matsuzaki, M., 1990, Earthquakes as self-organized critical phenomena, J. Geophys. Res.. 95, 6853-6860.

Kagan, Y. Y., 1994, Observational evidence for earthquakes as a nonlinear dynamic process, Physica D, 77, 160-192.

Kagan, Y. Y, and Knopoff, L., 1984, A stochastic model of earthquake occurrence, Proc. 8-th Int. Conf. Earthq. Eng., San Francisco, Calif,, vol. 1, 295-302.
Kanamori, H., 1978, Quantification of earthquakes, Nature, 271, 411-414.

Kanamori, H. and Anderson. D. L., 1975. Theoretical basic of some empirical relations in seimology, Bull, Seismol. Soc. Am., 65, 1073-1095.

Knopoff, L. and Kagan, Y. Y., 1977. Analysis of the theory of extremes as applied to earthquake problems, J. Geophys. Res., 82, 5647-5657.

Main, I. G., 1992, Earthquake scaling, Nature, 357, 27-28.

Main, I. G. and Burton, P. W., 1986, Long-term earthquake recurrence constrained by tectonic seismic moment release rates, Bull. Seismol. Soc. Am., 76, 297-304.

Marrett, R., 1994, Scaling of intraplate earthquake recurrence interval with fault length and implications for seismic hazard assessment, Geophys. Res. Lett., 21, 2637-2640.

Mogi, K., 1962, Magnitude-frequency relation for elastic shocks accompanying fractures of various materials and some related problems in earthquakes, Bull. Earthq. Res. Inst., Tokyo Univ., 40, 831-853.

Okal, A. and Romanowicz, B. A., 1994, On the variation of b-values with earthquake size, Phys. Earth Planet. Inter., 87, 55-76.

Pacheco, J. F., Scholz, C. H. and Sykes, L. R., 1992, Changes in frequencysize relationship from small to large earthquakes, Nature, $355,71-73$.

Rikitake, T., 1975, Dilatancy model and empirical formulas for an earthquake area, Pageoph, 113, 141-147.

Rikitake, T., 1986, Earthquake precursor phenomena: Database for prediction, Tokyo Univ. Press, Tokyo, 232 pp.

Romanowicz, B., 1992, Strike-slip earthquakes on quasi-vertical transcurrent faults: inferences for general scaling relations, Geophys. Res. Lett., 19 , 481-484.

Romanowicz, B. and Rundle, J. B., 1993, On scaling relations for large earthquakes, Bull. Seismol. Soc. Am., 83, 1294-1297.

Rundle, J. B., 1989, Derivation of the complete Gutenberg-Richter magnitude-frequency relation using the principle of scale invariance, J. Geophys. Res., 94, 12337-12342.

Scholz, C. H., 1968, The frequency-magnitude relation of microfracturing in rock and its relation to earthquakes, Bull. Seismol. Soc. Am., 58, 399. rock
415 .

Scholz, C. H., 1982, Scaling laws for large earthquakes: consequences fo physical models, Bull. Seismol. Soc. Am., 72, 1-14.

Sornette, D., Knopoff, L., Kagan, Y. Y. and Vanneste, C., 1996, Rank-ordering statistics of extremes events: Application to the distribution of large earthquakes, J. Geophys. Res., 101, 13883-13893.

Turcotte, D. L.. 1992, Fractals and chaos in geology and geophysics, 221 pp. Cambridge University Press, Cambridge.

Utsu, T., 1985, Seismology, Kyoritsu Press, Tokyo, 310 pp (in Japanese).

Motoji Ikeya, graduated from Dept. of Electronics Engineering, Osaka Uni. in 1963 and obtained Ph D in Nuclear Engineering. Research associates in Physics Dept., Uni. of North Carolina and fellow of the Humbodt Foundation from 19761978 at Uni. of Stuttgart. Professor of Osaka Uni. in Dept. of Physics in 1987 and of Earth and Space Sci. since 1991. Seismic electric signal, anomalous animal behavior are research interests after the Kobe Earthquake.

Qinghua Huang, Ph D student in Department of Earth and Space Sciences, Osaka University. Research interests include seismoelectricity, rupture physics and disaster prevention.
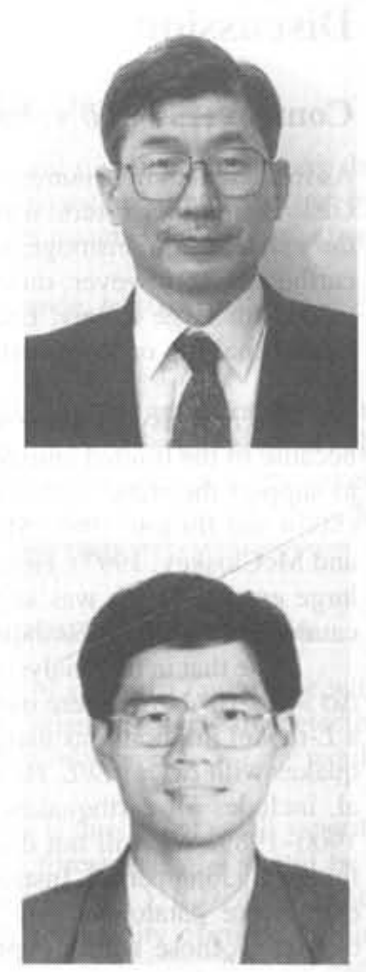